\title{
EDITORIAL
}

\section{COMPELLING EVIDENCE REVEALS THAT ORAL CHRONIC INFECTION AND ORAL INFLAMMATION GENERATE SYSTEMIC CONSEQUENCES}

The mouth is the gateway to our body. It represents $\sim 10 \%$ of human body volume; however, important functions rely on the mouth: eating, degustation, exploring the world as a child, chewing, digestion, tasting, biting, breathing through the mouth, vomiting, kissing, smiling, speaking, nonverbal communication, sex appeal, and social relations also depend on having a healthy and functional mouth. The teeth, tongue, gums, nerves, muscles, ligaments, veins, arteries, bones, connective tissue, and epithelia are organized in the complex structural array that constitutes the mouth. The mouth establishes anatomical relationships with other essential organs like the pharynx, the esophagus, the nose, the face, the ears, the orbits and, perhaps most important, the brain. The physiologic mouth boundary with the body is by far more complex. Just to mention that saliva is an extremely important defensive body fluid, released into the mouth by which innate and acquired immune mechanisms effectively defend the host against foreign insults. Saliva flow reduction indefectible results in increasing dental decay, periodontal disease, and oral ulcers. Therefore, the role of the mouth in improving and maintaining optimal general health is frequently underestimated. Most medical examinations of patients overlook the mouth and begin at the throat. To compensate for the oversight, the mouth now deserves all the possible attention from dentistry, with several dental specialties developed over the previous century. However, dentists fail to connect the mouth to the rest of the body. Advocating the importance of the health of the mouth in general health implies a huge challenge for medicine, dentistry, and people. For example, very few medical schools consider an oral medicine course as part of the medical curriculum and fewer dental schools teach internal medicine courses to their students to the misfortune of the profession and patient wellbeing. Perhaps, Flexner and Baltimore were right when they proposed around 1890 the convenience of regulating the curriculum in Medicine and Dentistry or fusing both professions. The Deans of Medical and Dental Schools must devise a strategy to correctly teach oral medicine to their students.
Fortunately, oral medicine/periodontal medicine, an ancient idea started by Miller in 1879 and adopted by Hunter in 1890, is now a recovered and robust field of knowledge. In 1989, two epidemiologic studies established the possible association among oral/ periodontal disease and myocardial infarction and stroke. Since then until now (November 2011) a PubMed search yielded more than 4000 articles published in this promising field. Compelling scientific evidence shows than diabetes, metabolic syndrome, cardiovascular disease, preeclampsia, premature delivery, low birth weight, respiratory disease, rheumatoid arthritis, osteoporosis, oral cancer, and pancreatic cancer may be linked to chronic oral infection and inflammation. Epidemiological evidence, instead, shows weak but consistent positive association of this link. There is also better space for the biological plausibility. Evidence recalled from animal experimentation and cell culture assays showed that some oral and periodontal microorganisms could even disrupt the production of nitric oxide in cultured endothelial cells. The DNA of some otherwise strictly oral microorganisms has been detected in atherosclerosis and $P$. gingivalis was viably recovered after artery plaque removal.

Two of the five most prevalent human diseases occur in the mouth. Dental caries and periodontal disease, including gingivitis affects the whole population during the life span. Both diseases are preventable by developing appropriate education policies aimed at all the population. Caries and periodontitis are treatable diseases by using secondary prevention and facilitating early treatment. It is widely accepted that oral infection and mouth microbial overgrowth triggers chronic local inflammation and also that periodontal disease generates ulceration of the crevicular epithelia facilitating bacteremia. Still, it is a matter of debate if untreated chronic gingivitis or periodontitis leads to systemic inflammation. However, there is a good chance that via continuous bacteremia and disseminating proinflammatory cytokines developed over the time a low-grade inflammation with systemic consequences might be established. Paraskevas et al. (2008), in a systematic 
review, demonstrated that untreated periodontal disease significantly increases hs-PCR, and treatment of periodontitis reduced its levels.

Living with microbes is a cruel reality. Only $10 \%$ of the cells in the human body are human, 90\% are procaryotic cells. Fortunately, most of the relationships between host mammalian cells and bacteria are symbiotic. The human mouth is colonized from birth to death by different microbes organized in biofilms constituted mainly by bacteria and Candida species; however, it may also harbor some parasites and viruses. Biofilms are recalcitrant to removal from hard or mucous surfaces. Regular mechanical disruption of oral biofilm is an important practice to avoid overgrowth of certain species related to oral mucosal/gingival inflammation. Most microbial composition in individuals originates from their mothers and it is acquired early in life through breastfeeding, kissing, and food sharing. Saliva is the main vehicle for microbial transmission. The father, close relatives, and baby sitter possibly contributed in part to this early microbial acquisition in a child's mouth. Finally, close interaction with peers and contact with contaminated objects in daycare centers, sexual initiation, kissing, and cohabitation with a partner are some of the final sources for late microbial colonization in individuals. Transient and permanent microbial colonization completes the eight microbial taxa and 150 to 1000 species that human harbor in their mouths. A mouth offers several ecological niches and provides diverse environments for aerobic, facultative, and anaerobic microbes. The host's cell receptors interact with microbial adhesins and fimbriae and also facilitate entry ports for viruses. Tooth eruption also facilitates biofilm organization and maturation and subgingival biofilm organization. It is necessary to recognize that a coevolution between the host's structures and the microbes had been developed over time. Microbes should not harm the host. Microbial dysbiosis is caused by microbial overgrowth of a pathogenic species and suppression of beneficial species, and/or over-stimulation of host immune response is believed to be the cause of oral and many microbialinduced diseases. To maintain good oral health, regular removal of oral biofilm is a mandatory issue. But, how do we keep oral biofilm at a level related to oral health? Regular brushing of the teeth and tongue and interdental flossing is a must, accompanied by the use of antibacterial toothpastes and oral rinse. Every day, proper oral hygiene practice controls most oral biofilmrelated disease. Thus, education of oral hygiene measures is necessary to create good habits in individuals starting from six months of age (from the apparition of baby teeth) with help from parents, reinforced by teachers at daycare centers and school, dentists and dental hygienists. Reducing intake of food rich in sugar and using fluoride in water and tooth sealants, diminish the risk of dental decay. If biofilm is controlled over the time, gingivitis and periodontal disease may also decrease their prevalence rates in the population. When considering the impact of keeping good oral health on reducing its influence upon chronic inflammation in systemic disease, the economic impact -yet to be determined- may be significant in terms of public health policies.

In conclusion, dentistry needs to become be more medically oriented and medicine more orally oriented. Chronic inflammation seems to harm the body. Oral biofilm requires continuous efforts from patients for removal. Oral health policies must be promoted at individual and population levels. The mouth is connected to the body and its diseases also affect general health.

\section{BIBLIOGRAPHY}

D’Aiuto F, Nibali L, Parkar M, Patel K, Suvan J, Donos N. Oxidative strees, systemic inflammation, and severe periodontitis. J Dent Res. 2010; 89: 1241-6.

Haraszthy VI, Zambon JJ, Trevisan M, Zeid M, Genco RJ. Identification of periodontal pathogens in atheromatous plaques. J Periodontol. 2000; 71:1554-60.

Mattila KJ, Nieminen MS, Valtonen VV, Rasi VP, Kesaniemi YA, Syrjala SL, et al. Association between dental health and acute myocardial infarction. BMJ 1989; 298: 779-81.

Paraskevas S, Huizinga JD, Loos BG. A systematic review and meta-analyses on C-reactive protein in relation to periodontitis. J Clin Periodontol 2008; 35: 277-90.

Ramírez JH, Arce R, Contreras A. Why must physicians know about oral diseases? Teach Learn Med 2010; 22:148-55.

Adolfo Contreras, OD, MSc, $P h D$ Full Professor and Professor Emeritus School of Dentistry, Faculty of Health Universidad del Valle, Cali, Colombia e-mail: adolfoco@yahoo.com 